\title{
LYMAN THE FAR ULTRAVIOLET SPECTROSCOPIC EXPLORER
}

\author{
WARREN MOOS \\ The Johns Hopkins University, Department of Physics and Astronomy, \\ Homewood Campus, Baltimore, Maryland 21218
}

\begin{abstract}
The Lyman Far Ultraviolet Spectroscopic Explorer mission will obtain high resolution spectra ( $R 30,000$ ) in the $912-1200 \AA$ region with sufficient sensitivity to study faint sources throughout the galaxy and at large extragalactic distances. This spectral region provides unique tools for solving problems in cosmology (e.g. deuterium), galactic structure and evolution (e.g. O VI and $\mathrm{H}_{2}$ ), stellar evolution (e.g. O VI) and planetary science (e.g. $\left.\mathrm{H}_{2}\right)$. Recent advances in optical and detector technology, which make this goal possible with a moderate size experimental package, also enable the spectral coverage to extend down to $100 \AA$ with good sensitivity and only a minimal increase in complexity. Thus a secondary goal is to cover the $100-912 \AA$ region with moderate spectral resolution. In 1989, following a Phase A study of the mission concept, NASA selected the mission for Phase B study in 1989. Both Canada and the United Kingdom are participating in the definition and development of the mission.
\end{abstract}

\section{Mission Overview}

The Lyman Far Ultraviolet Spectroscopic Explorer (FUSE) completed its Phase A study in June of 1989. The Phase B Definition Study will begin in the summer of 1990. Phases C/D are expected to start in July of 1992 and the launch is planned for 1997. This paper is based on the Phase A study and Table I lists the scientific personnel associated with the study. Table II is a brief summary of the mission. Additional details are given in the final report for the study (Moos et al. 1989).

The FUSE instrument consists of a $70 \mathrm{~cm}$ Wolter type II glancing incidence telescope with 1 arcsec image quality feeding a spectrograph. This spectrograph has several channels which provide high resolving power on the order of 30,000 from the Hubble Space Telescope limit at near $1200 \AA$ down to the limit set by the photoionization continuum of atomic hydrogen at $912 \AA$. In addition other channels in the spectrograph will provide moderate resolving power down to $100 \AA$. Most of the channels on the instrument are astigmatic and permit imaging along the slit to remove background or study extended objects. The sensitivity is quite high and as a result is appropriate for bridging the gap between the Hubble Space Telescope (HST) with a cut-off near $1200 \AA$ and the Advanced X-ray Astrophysics Facility (AXAF) with an upper limit near $100 \AA$. Figure 1 shows the estimated limiting sensitivity of the FUSE instrument in comparison with several other missions.

The Canadian Space Agency and the British National Space Center are cooperating with NASA in the definition and development of the FUSE mission. Canada will supply the baffle system for the telescope and cooperate with the United Kingdom in developing the fine error sensor. In addition to development of the fine error sensor, the United Kingdom will also supply the focal plane assembly, the main electronics box and the associated software.

Y. Kondo (ed.), Observatories in Earth Orbit and Beyond, 171-176.

(C) 1990 Kluwer Academic Publishers. Printed in The Netherlands. 
TABLE I

Scientific Personnel for Phase A Study

Co-Investigators

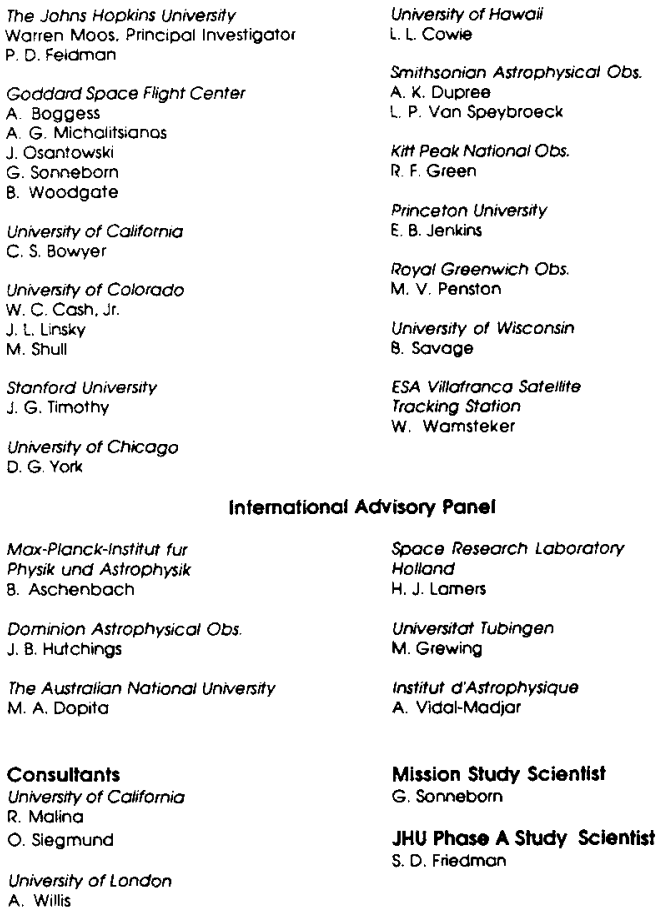

\section{Scientific Goals}

The Lyman FUSE mission has a powerful and unique set of tools for understanding astrophysical problems. A number of astrophysically important species such as deuterium, molecular hydrogen, helium, $\mathrm{O}^{+}$to $\mathrm{O}^{5+}, \mathrm{Ne}$ to $\mathrm{Ne}^{5+}, \mathrm{A}, \mathrm{S}^{3+}$ to $\mathrm{S}^{12+}$ and many others have their strongest transitions in this spectral region. The number of interstellar absorption transitions increases dramatically below $1200 \AA$. Emission and absorption transitions in this spectral region come from species at very different temperatures ranging from molecules to highly ionized species such as Fe XXIV. Ratios of emission intensities provide electron densities over the range of $10^{6}$ to $10^{14}$ electrons $/ \mathrm{cm}^{3}$. The high spectral resolution will permit the measurement of gas velocities as small as a kilometer per second. Finally the high sensitivity permits the study of new classes of objects which were not accessible to the Copernicus mission or other missions which studied this spectral region. The ability to study white dwarfs will permit accurate measurements of interstellar species in the near interstellar medium. Extra-galactic sources such as active galactic nuclei will be used to study the gas at the very edge in the halo of the galaxy and between galaxies out to large distances. It is worth noting that the Copernicus mission was able 


\section{Bridging the Gap}

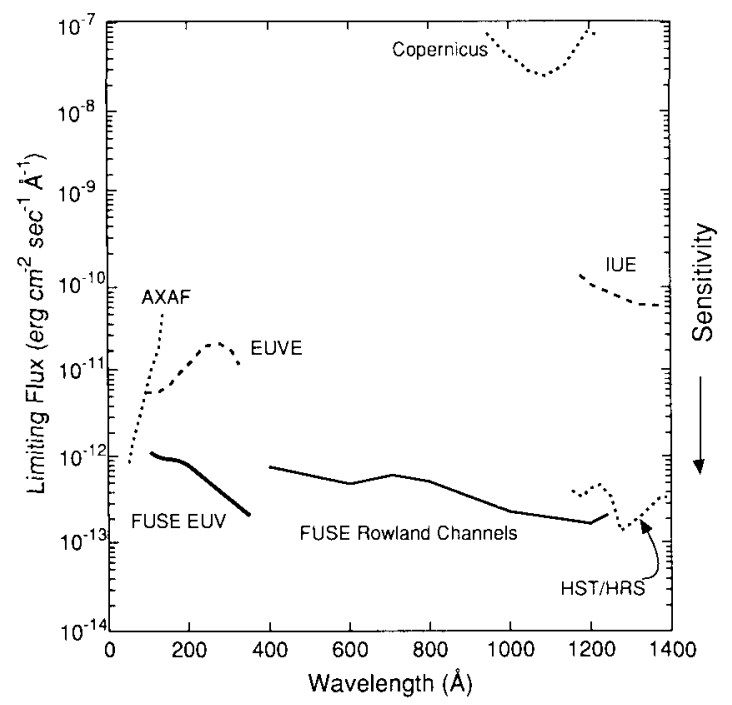

Fig. 1. The sensitivity of Lyman FUSE compared with other UV and EUV missions. The most sensitive instruments are at the bottom of the graph. An integration time of 1800 seconds and 100 detected photons per resolution element are assumed. The HST/HRS flux limits were computed for coverage of a $120 \AA$ section of the spectrum, equal to the spectral range of each of the FUSE high-resolution Rowland channels. The Copernicus sensitivity is off the top of the graph and is displayed only to show the shape of the response.

to measure the deuterium to hydrogen ratio in approximately a dozen hot stars within about 1000 parsecs of the sun. In addition a similar number of stars have been measured using the chromospheric Ly $\alpha$ line using both the Copernicus and IUE satellites (Boesgaard and Steigman 1985). As a result, reliable measurements of this cosmologically important number exist only for gas clouds in a small vicinity of our spiral arm.

The Phase A Study team examined the areas where the FUSE mission would make the most important advances and concluded that there were three major areas. First, it will expand our understanding of the early universe by providing reliable measurements of big bang light element nuclear synthesis and also by measuring the temperature of the gas between galaxies. Second, the mission will also be an important tool for understanding the evolution and fundamental processes in galaxies. Measurements will range from the hot gas in disks and halos of galaxies to examining supernova and their remnants which feed much of this hot gas into the galaxy. Third, the mission will provide fundamental insight into a number of problems associated with the evolution of stars and planetary systems. In particular it is expected that accretion processes, winds and magnetic activity in solar type stars, studies of primordial abundances in the solar system, and planetary atmospheric excitation processes will be important.

Figure 2 shows a simulated spectrum for a hot object behind a cloud with a 


\section{Determining the Deuterium Abundance}

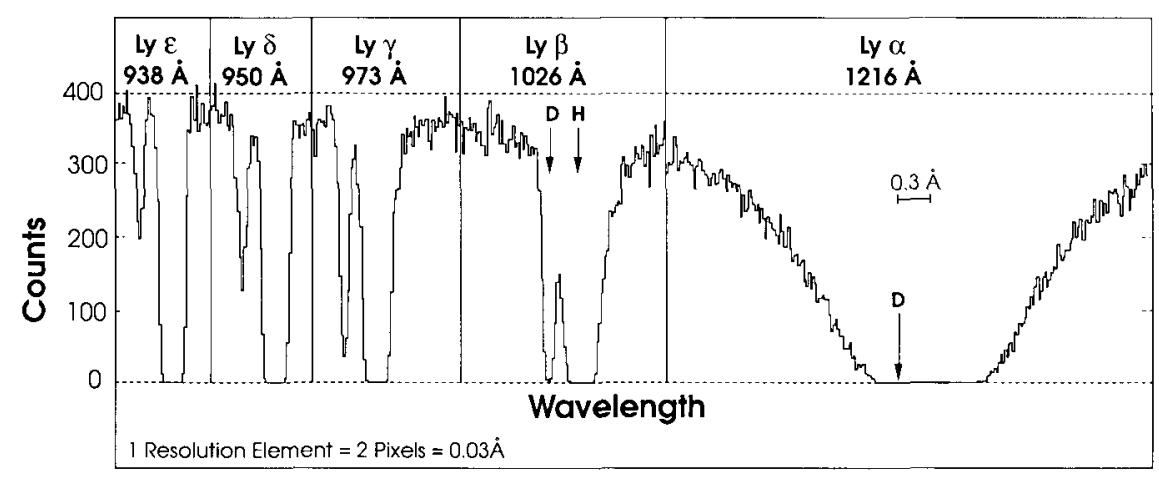

Fig. 2. Absorption line spectrum showing the splitting of the $H$ (broad) and D (narrow) lines. This simulated spectrum corresponds to $10^{5} \mathrm{sec}$ integration using an approximately 16th mag QSO as a background source. An abundance ratio of $\mathrm{D} / \mathrm{H}=2 \times 10^{-5}$ and a hydrogen column density of $\mathrm{N}_{\mathrm{H}}=3 \times 10^{19} \mathrm{~cm}^{-2}$ has been assumed.

deuterium abundance of 2 parts in $10^{5}$. It is clear that near $1200 \AA$ it is not possible to make reliable measurements. In fact one must go shortward of even Ly $\beta$ and well into the Lyman series before one could obtain lines which are not heavily saturated. Thus, the full range of the FUSE mission is necessary to provide reliable measurements of deuterium. Deuterium is thought to be a critical indicator of the density at about 100 seconds in the Big Bang. As a consequence, it will be an important diagnostic of the baryon density then and now and will help in understanding the missing mass issue. However, in order to determine the primordial value, it is important to understand the effect of possible processing of the primordial material. The sensitivity of the instrument will enable FUSE to study the deuterium abundance in many environments with different evolutionary histories both at a number of locations in our galaxy, in nearby galaxies, and in the gas between galaxies out to very large distances.

The FUSE mission will provide new information on the location of the hot gas in the disks and halos of galaxies. The very important lithium-like oxygen ion OVI can be studied only in this spectral region. As mentioned previously, the sensitivity of FUSE will make it possible to examine the halo gas in absorption using objects outside the galaxy as light sources. It is important to note that this mission will be important for studying supernova. It is quite possible that the interaction of supernova $1987 \mathrm{a}$ as it expands into the interstellar medium with that medium will lead to a sharp increase in the ultraviolet emissions in the late $90 \mathrm{~s}$. In addition, the sensitivity of the instrument will be such that several extragalactic supernova that are discovered each year using routine patrols can be studied without disrupting the regular observing program.

Because the ions which emit in this spectral region exist over a wide range 


\title{
TABLE II
}

Lyman FUSE Mission Summary

\begin{abstract}
Telescope: $70 \mathrm{~cm}$ diameter and 7 meter focal length. Wolter type II glancing incidence with gold overcoat. One arcsec image diameter image.
\end{abstract}

Spectroscopic Capabilities: High spectral resolution. Three channels cover $910-1250 \dot{A}$ with $R=30,000$ at 1 arcsec width. Imaging along 45 arcsec long slits divided into 15 arcsec segments with widths of $0.5,1.0$ and 3.0 arcsec.

Planetary and survey grating. $400-1600 \AA$. Imaging along 45 arcsec long slit divided into a 30 arcsec by 4 arcsec segment $(R=1000$ ) and a 15 arcsec by 1 orcsec segment $(R=4000)$.

EUV grating. $100-350 \AA$ with $R=300$ and 800 . Limited imaging along slit.

Spacecraft and Orbit: Explorer Bus (MMS spacecraft) in $\sim 500 \mathrm{~km}$ orbit with $28^{\circ}$ inclination. Three year mission.

Tracking: Explorer Bus Inertial Reference Units and Star Trackers with updates by Fine Error Sensor in telescope focal plane. Pointing uncertainty $\sim 0.3$ arcsec. FES field of view is 7 arcmin by 7 arcmin. Dynamic range is $17 \mathrm{~m}$ to $-2 \mathrm{~m}$ for point ( 1 arcsec) sources. Solar avoidance angle is $43^{\circ}$.

Guest Observer Program: Most of the observing time available to the scientific community through a Guest Observer program similar to those of IUE and HSI

of temperatures, this mission will be extremely important for studying the outer atmospheres of late type stars. The fundamental problems to be addressed include whether stellar coronae exist at all temperatures, and in fact how do such solar type stars lose mass. The wavelength region covered by the FUSE instrument includes features from species which exist at very different temperatures. Thus, emission measures will be determined for the stellar plasma from a few thousand degrees to several tens of millions of degrees.

\section{Status of the Technology}

As part of the Phase A study, the Science Team constructed a detailed candidate observing plan. For the instrument parameters of Table II, the observing plan would require about one year of observing time for successful completion. Assuming a typical efficiency for lower earth orbit, a three-year mission will be necessary to accomplish the baseline science goals. The instrumentation technology is now at a point where this mission is practical. The telescope performance and optical fabrication errors and related manufacturing problems have been analyzed in detail by industrial firms. A design study has demonstrated the feasibility of a fine error sensor with a $7 \times 7$ arcminute field of view. The development of silicon carbide coatings with normal incidence reflectivities greater than $30 \%$ below $1000 \AA$ will give high transmission down to well below $900 \AA$. A prototype of the distorted 
ellipsoid surface necessary for astigmatic imaging in the Rowland mount has been constructed (Cash 1989). The EUV gratings and detectors are based on the technology developed for the EUV mission: The FUV detectors are based on detectors presently under development for STIS, SOHO and EUVE. Finally, the very careful and detailed modelling of the mechanical and thermal stability of the structure has shown that it can be achieved without heroic efforts. The Explorer platform which is being constructed for the EUVE and XTE mission is baselined for this mission. The instrument has been designed so that it will be dual adaptable to either a shuttle or expendable launch vehicle, thus providing two paths into space.

Mission operations will build on the EUVE and XTE experience and software and will use the facilities developed for those missions. The Science Operations Center will be located at the Goddard Space Flight Center and a Guest Investigator program will be allocated about $90 \%$ of the observing time.

\section{Summary}

The Lyman FUSE mission will have a major impact on both astrophysics and science as a whole by providing new spectroscopic measurements of unparalled sensitivity in the $100 \AA$ to $1200 \AA$ region. Understanding of the scientific problems, necessary data and the required technical capabilities of the instrumentation are at a point where large steps forward are to be expected for many important scientific problems. Finally, of extreme importance, the technology is mature and ready for application in a space environment.

\section{References}

Boesgaard, A.M. and Steigman, G.: 1985, Palo Alto: Annual Reviews 23, 319

Cash, W.C., Jr., 1989, private communication

Moos, H.W. et al.: 1989, Lyman The Far Ultraviolet Spectroscopic Explorer: Phase A Study Final Report 